Spatial Network Big Databases 
KwangSoo Yang · Shashi Shekhar

\section{Spatial Network Big Databases}

Queries and Storage Methods

粤 Springer 
KwangSoo Yang

Computer Science Department

Florida Atlantic University

Boca Raton, FL

USA

\author{
Shashi Shekhar \\ Department of Computer Science \\ and Engineering \\ University of Minnesota \\ Minneapolis, MN \\ USA
}

\section{ISBN 978-3-319-56656-6 \\ ISBN 978-3-319-56657-3 (eBook) \\ DOI 10.1007/978-3-319-56657-3}

Library of Congress Control Number: 2017937267

(C) Springer International Publishing AG 2017

This work is subject to copyright. All rights are reserved by the Publisher, whether the whole or part of the material is concerned, specifically the rights of translation, reprinting, reuse of illustrations, recitation, broadcasting, reproduction on microfilms or in any other physical way, and transmission or information storage and retrieval, electronic adaptation, computer software, or by similar or dissimilar methodology now known or hereafter developed.

The use of general descriptive names, registered names, trademarks, service marks, etc. in this publication does not imply, even in the absence of a specific statement, that such names are exempt from the relevant protective laws and regulations and therefore free for general use.

The publisher, the authors and the editors are safe to assume that the advice and information in this book are believed to be true and accurate at the date of publication. Neither the publisher nor the authors or the editors give a warranty, express or implied, with respect to the material contained herein or for any errors or omissions that may have been made. The publisher remains neutral with regard to jurisdictional claims in published maps and institutional affiliations.

Printed on acid-free paper

This Springer imprint is published by Springer Nature

The registered company is Springer International Publishing AG

The registered company address is: Gewerbestrasse 11, 6330 Cham, Switzerland 


\section{Preface}

Spatial Network Big Data (SNBD) refers to spatial network datasets whose size, variety, or update rate exceeds the capacity of commonly used spatial network computing and spatial network database technologies to learn, manage, and process with reasonable effort. SNBD has the potential to transform society via nextgeneration routing services, emergency and disaster response, and discovery of potentially useful patterns embedded in these datasets. The use of SNBD is rapidly expanding into the transportation arena to improve the management and security of transportation infrastructure and enable data-driven decision-making. However, there are significant challenges to the use of SNBD because current methods, models, and algorithms do not always scale and/or perform well when storing, managing, and analyzing large volumes of data. Interestingly, most of the SNBD collected today are not used at all, and data that are used are not fully exploited. In addition, SNBD datasets tend to be used mostly for real-time control or anomaly detection, rather than optimization or prediction based on historical data, which is where their greatest value lies. Investigating novel SNBD storage and processing platforms, such as database systems and data analytics tools, is critical to realize the full value of SNBD.

Developing Spatial Network Big Database Systems (SNBDS) requires overcoming three key challenges. First, it requires new data models to represent the complex and interrelated structure of SNBD. Second, fully exploiting SNBD requires scalable query processing and optimization methods, which are currently lacking. Finally, SNBDS require I/O efficient storage and access methods that leverage scalability and efficiency of big data query processing. These challenges lead us to rethink both existing theories and models. This book presents a collection of concepts, algorithms, and techniques that effectively harness the power of SNBD. Reading this book is a first step toward understanding the immense challenges and novel applications of SNBD database systems. This book is organized in seven chapters. After reviewing some preliminaries in Chap. 1, we 
introduce basic graph algorithms in Chap. 2. Chapters 3-5 formally model spatial network query problems and explore algorithms that minimize the computational cost for query processing. Chapter 6 introduces strategies to develop I/O efficient storage and access methods. Chapter 7 summarizes the book's major themes.

Boca Raton, FL, USA

KwangSoo Yang

January 2017 


\section{Acknowledgements}

I would like to express my sincere appreciation and thanks to my advisor Prof. Shashi Shekhar, Department of Computer Science, University of Minnesota. His enthusiasm, inspiration, and great efforts helped me to grow as a research scientist. I would like to thank Springer for their advice and support to publish this book. I would like to express my gratitude to the University of Minnesota Spatial Computing Research Group for their brilliant comments and suggestions. I would like to thank Kim Koffolt for improving the readability of this book. Lastly, I thank my mother, sister, brother, and the Lundberg family for everything they have done for me. 


\section{Contents}

1 Spatial Network Big Databases: An Introduction. . . . . . . . . . . 1

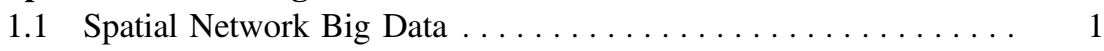

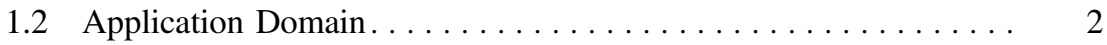

1.3 Spatial Network Big Database Management Systems. . . . . . . . . . 2

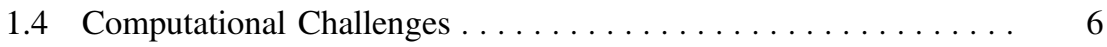

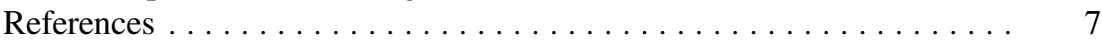

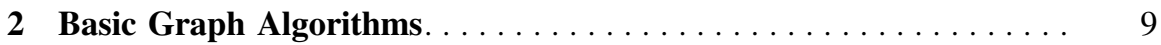

2.1 A Brief Introduction to Graph Theory ................ 9

2.2 Network Representations . . . . . . . . . . . . . . . . . . 9

2.2.1 Node-Node Adjacency Matrix . . . . . . . . . . . . . . . 10

2.2.2 Node-Edge Incidence Matrix . . . . . . . . . . . . . . . . . . . . . . . . . . 10

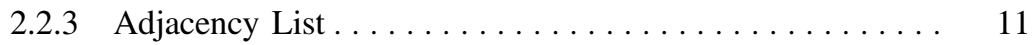

2.2 .4 Forward Star. . . . . . . . . . . . . . . . . . . . . . . 12

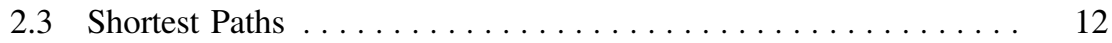

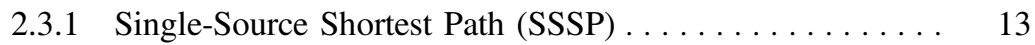

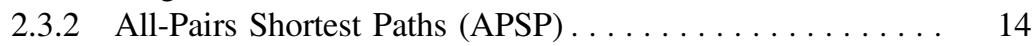

2.4 Block Decomposition. . . . . . . . . . . . . . . . . 15

2.5 Maximum Network Flow. . . . . . . . . . . . . . . . . . 16

2.5.1 Augmenting-Path Algorithm............... 16

2.5.2 Push-Relabel Algorithm . . . . . . . . . . . . . . . . . 18

2.6 Bipartite Weighted Matching . . . . . . . . . . . . . . . . 19

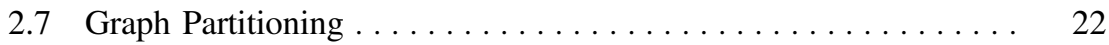

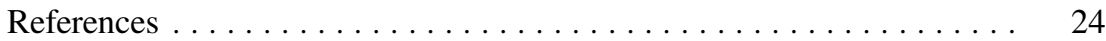

3 Capacity Constrained Network Voronoi Diagrams . . . . . . . . . 27

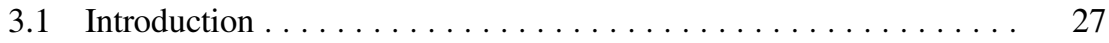

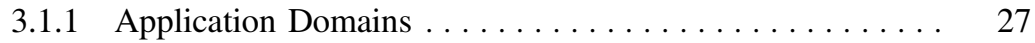

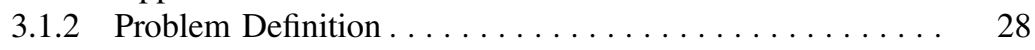

3.1.3 Problem Hardness. . . . . . . . . . . . . . . . . . 29 
3.1.4 Literature Review. . . . . . . . . . . . . . . . . . 30

3.1.5 Outline of the Chapter ................. 31

3.2 Algorithms for Capacity Constrained Network Voronoi Diagram............................. 32

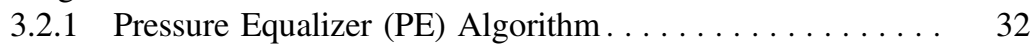

3.2.2 PE-BTCC Algorithm . . . . . . . . . . . . . . . . . 35

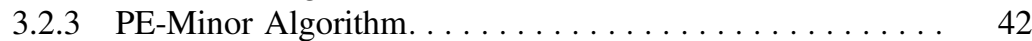

3.3 Case Study with Brooklyn, NY Road Network ........... 43

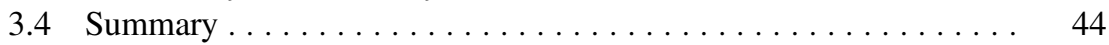

References .............................. 44

4 Distance-Constrained k Spatial Sub-networks . . . . . . . . . . . 47

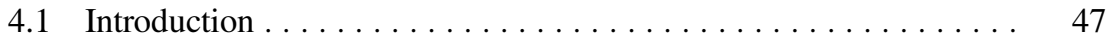

$4.1 .1 \quad$ Application Domain ...................... 47

4.1 .2 Problem Definition..................... 48

4.1 .3 Problem Hardness. . . . . . . . . . . . . . . . . . . . . . . 49

4.1 .4 Literature Review. . . . . . . . . . . . . . . . . . . . . . 49

4.1.5 Outline of the Chapter ................. 50

4.2 Algorithm for Distance-Constrained k Spatial Sub-networks .... . 50

4.3 Case Study with Chicago Road Network ................ 54

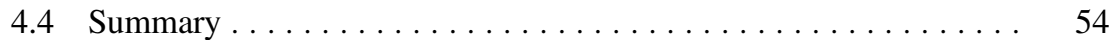

References ............................ 55

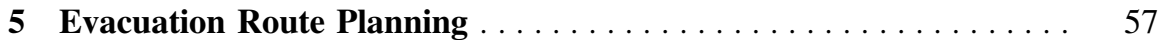

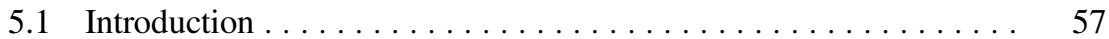

$5.1 .1 \quad$ Application Domain . . . . . . . . . . . . . . . . . 57

5.1 .2 Problem Definition . . . . . . . . . . . . . . . 58

5.1 .3 Literature Review....................... 58

5.1 .4 Outline of the Chapter .................. 59

5.2 Algorithms for Evacuation Route Planning ............ 60

5.2.1 Capacity Constrained Route Planner Algorithm ......... 60

5.2.2 Dartboard Network Cuts for Evacuation Route

Planning Algorithm ....................... 62

5.3 Experimental Analysis ....................... 67

5.3.1 Experiment Design . . . . . . . . . . . . . . . . 68

5.3.2 Experimental Results and Analysis. . . . . . . . . . . . 69

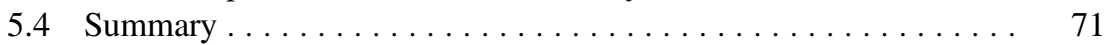

References ............................ 71

6 Storage Schemes for Spatio-Temporal Network Datasets . . . . . . . 73

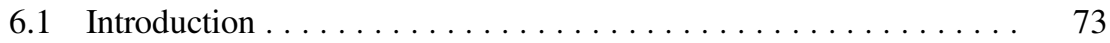

6.1 .1 Application Domains ..................... 73

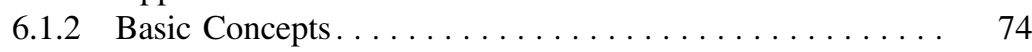

6.1.3 Problem Statement ..................... 77 
6.1.4 Literature Review . . . . . . . . . . . . . . . . . . 77

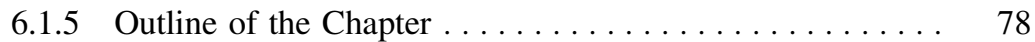

6.2 Lagrangian-Connectivity Partitioning Approaches for SSTN . . . . 79

6.2.1 LCP-G1S for LGetOneSuccessor $($. . . . . . . . . . . . . . 79

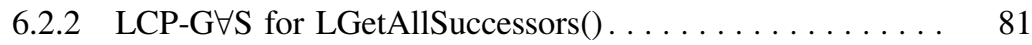

6.2 .3 Algorithm for $\mathrm{LCP}-\mathrm{G} \forall \mathrm{S} \ldots \ldots \ldots \ldots \ldots \ldots \ldots \ldots$

6.3 Cost Models. . . . . . . . . . . . . . . . . . . . . . . . . 90

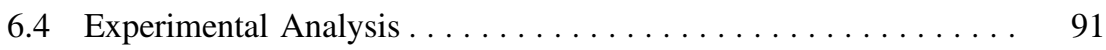

6.4.1 Experimental Design . . . . . . . . . . . . . . . 91

6.4.2 Experimental Results and Analysis.............. 93

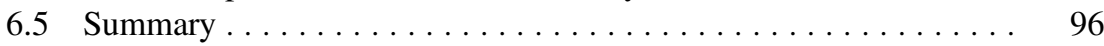

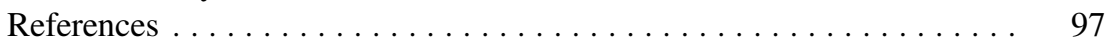

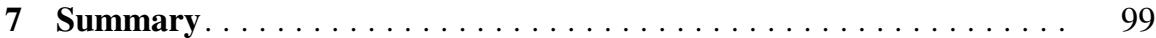

7.1 Capacity Constrained Network Voronoi Diagram . . . . . . . . . . . 100

7.2 Distance-Constrained $k$ Spatial Sub-networks. . . . . . . . . . . . . 100

7.3 Evacuation Route Planning . . . . . . . . . . . . . . . . . 100

7.4 Storage Schemes for Spatio-Temporal Network Datasets. . . . . . . 101 